\title{
The Feeding Behavior of Dogs Correlates with their Responses to Commands
}

\author{
Yuta OKAMOTO ${ }^{1)}$, Nobuyo OHTANI ${ }^{2)}$, Hidehiko UCHIYAMA ${ }^{2)}$ and Mitsuaki OHTA ${ }^{2)}$ \\ 1)Animal Life Solutions Co., Ltd., 16-6 Ibukino, Midoriku, Yokohama, Kanagawa 226-0028 and 2)Department of Animal Science and \\ Biotechnology, Azabu University School of Veterinary Medicine, 1-17-71 Fuchinobe, Sagamihara, Kanagawa 229-8501, Japan
}

(Received 19 January 2009/Accepted 25 August 2009)

\begin{abstract}
Motivation is one of the most important factors in dog training. To generate motivation, people use various reinforcer mechanisms. In particular, many pet owners use food because it is simple and convenient. The aim of this study was to investigate the association between dogs' level of interest in food and their responsiveness to commands. Thirty-four dogs were divided into three groups based on their feeding patterns (Fast, Slow, and Leftover). The fast group $(n=15)$ had the highest interest in food and showed a high response to commands when food was used as a reinforcer, rather than praise/stroking. The leftover group $(\mathrm{n}=6)$ showed the least interest in food and had a low reaction to commands, regardless of the reinforcer. In contrast, the slow group ( $\mathrm{n}=13$ ) had modest interest in food and maintained the same response to commands regardless of the reinforcer. Results of this study indicate that dogs' feeding patterns are indicative of their level of interest in food, and may be useful in determining the optimal training reinforcer. This can help dog owners improve their relationships with their dogs.
\end{abstract}

KEY WORDS: behavior, canine, commands, feeding, reinforcer.

J. Vet. Med. Sci. 71(12): 1617-1621, 2009

Operant conditioning has been popularly used in dog training to teach an animal that its response to a command has consequences [8]. The observation that the consequences of a behavior can determine whether the behavior will occur again led Thorndike to the 'Law of Effect' which states that if a consequence is pleasant, the preceding behavior becomes more likely. If a consequence is unpleasant, the preceding behavior becomes less likely [11]. For example, if a dog sits in response to the command 'sit' and then receives a treat, and then this sequence is repeated, the dog will establish a link between the command, the response, and the treat. In this case, the dog's response to the command has been positively reinforced by the treat [8]. There are many types of reinforcer for dogs such as food, toys and praise. Many dog owners have used food as reinforcer because of its convenience.

When learning, motivation is one of the most important factors that influences learned behavior. In other words, learning might not occur without motivation [8]. Once a behavior has been learned, it may not be performed unless the animal is motivated to respond. A reinforcer serves to provide motivation. There is variation among individuals in the effectiveness of a reinforcer; for instance, some dogs are motivated to learn with food, while others would prefer toys.

The dog origin from wolves is well established from genetic as well as behavioral and morphological data $[2,14$, 15]. The digestive tract of wolf is specialized and able to handle large quantities of food in a single meal - a distinct advantage in a competitive feeding situation [12]. Although

\footnotetext{
* Correspondence to: Ohtani, N., Laboratory of Effective Animals for Human Health, Azabu University School of Veterinary Medicine, 1-17-71 Fuchinobe, Sagamihara, Kanagawa 2298501, Japan.

e-mail: ohtani@azabu-u.ac.jp
}

the domestication process has altered the feeding behavior of dog, some breeds still demonstrate a remarkable agility to gorge, and will eat exceptionally large quantities of food whenever it is available [12]. This characteristic may also be influenced by gender, age, breed and dietary factors. Several breeds of dogs have a reputation for being able to consume large meals very rapidly, and it is possible that this is the result of competitive feeding in the wolves from which they were domesticated [1]. Certain breeds that have a high propensity to gain weight, such as Cocker Spaniel, Labrador Retriever, Dalmatian, Dachshund, Golden Retriever, and Shetland Sheepdog may have a particularly high interest in food $[4,7]$. A dog has high interest in food and which has been trained using food as a reinforcer, has difficulty in switching to secondary reinforcer. Such dog sometimes rejects the owner's command when it knows its owner doesn't have food. While food is the convenient reinforcer, it is difficult to build good relationship with the dog and control it if the owner doesn't understand his/her dog's level of interest in food.

The aim of this study was to examine the relationship between dogs' level of interest in food and their response to the commands, as related to feeding pattern. Such a correlation would suggest that identification of a dog's feeding pattern may contribute to the selection of a suitable reinforcer and training strategy.

\section{MATERIALS AND METHODS}

Subjects: The subjects were 34 healthy dogs of 21 different breeds (18 males and 16 females): 23 dogs were studied at the World Ranch in Osaka, Japan, and 11 dogs were studied at the Murase Dogs Training Center in Kanagawa, Japan (Table 1). All of the dogs were sexually intact and 12-96 months of age (mean $50.7 \pm 4.4$ months, excepting one dog 
Table 1. Profile of the subjects

\begin{tabular}{|c|c|c|c|}
\hline Breed & Sex & $\begin{array}{c}\text { Age } \\
\text { (months) }\end{array}$ & Weight \\
\hline \multicolumn{4}{|l|}{ Fast group } \\
\hline Brittany & Male & 18 & 15 \\
\hline Brittany & Male & 18 & 15 \\
\hline Dalmatian & Male & 95 & 29 \\
\hline Dobermann & Female & 72 & 31 \\
\hline Golden Retriever & Female & 60 & 27 \\
\hline Golden Retriever & Male & 38 & 22.5 \\
\hline Labrador Retriever & Male & 36 & 29 \\
\hline Labrador Retriever & Male & 36 & 31 \\
\hline Labrador Retriever & Female & 96 & 23 \\
\hline Labrador Retriever & Female & 12 & 23 \\
\hline Labrador Retriever & Male & 71 & 31 \\
\hline Labrador Retriever & Male & 46 & 25.5 \\
\hline Labrador Retriever & Male & 39 & 24 \\
\hline Newfoundland & Female & 66 & 36 \\
\hline Standard Poodle & Male & 13 & 21 \\
\hline \multicolumn{4}{|l|}{ Slow group } \\
\hline American Cocker Spaniel & Female & 53 & 10 \\
\hline Australian Shepherd Dog & Male & UN & 17 \\
\hline Basset Hound & Female & 48 & 20 \\
\hline Border Collie & Female & 38 & 16 \\
\hline Clumber Spaniel & Female & 55 & 19 \\
\hline Flat-coated Retriever & Male & 78 & 24 \\
\hline Flat-coated Retriever & Female & 41 & 21 \\
\hline German Shepherd Dog & Female & 60 & 26 \\
\hline German Shepherd Dog & Female & 72 & 25 \\
\hline German Shepherd Dog & Male & 19 & 28 \\
\hline Golden Retriever & Female & 37 & 22 \\
\hline Rough Collie & Male & 19 & 25 \\
\hline Weimaraner & Female & 91 & 21 \\
\hline \multicolumn{4}{|l|}{ Leftover group } \\
\hline American Cocker Spaniel & Male & 77 & 8 \\
\hline English Cocker Spaniel & Male & 58 & 9 \\
\hline Greyhound & Female & 96 & 23 \\
\hline Jack Russell Terrier & Male & 17 & 5.5 \\
\hline Pyrenean Mountain Dog & Female & 46 & 23.5 \\
\hline Vizsla & Male & 52 & 21 \\
\hline
\end{tabular}

whose age was unknown). They were housed in individual metal cages and provided with commercial dog food (Adult Maintenance, Nutro Products Inc., CA, U.S.A.), according to the industrial recommendation. Although their background (place of their birth, duration until they came to facility, etc.) varied, all of them were kept at each facility for at least one year. All dogs had been taught basic commands, such as "sit" and "down", by the staff at each facility using the positive reinforcement technique $[3,9,10,13]$ and their own food as the primary reinforcer and praise/stroking as the secondary reinforcer. All of the procedures were approved by the Animal Experiments Ethics Committee of Azabu University.

Classification of the dogs according to their feeding pattern: To classify the dogs, we recorded their normal feeding behavior for ten minutes using a digital video camera (GSCR60, Toshiba, Japan). By measuring weight of food and duration of feeding, the feeding speed $(\mathrm{g} / \mathrm{sec})$ and the amount of food consumed per mouthful (g/chew) were calculated. All dogs were divided into 2 groups; those who consumed their food completely and those who only partially consumed their food (Leftover). The dogs who completely consumed their food were then divided into 2 groups according to whether they chewed every mouthful more than once (Slow) or not (Fast). The dogs were therefore divided into the three groups according to feeding pattern, Leftover, Slow and Fast.

Experimental procedure: The outline of the experiment is shown in Fig. 1. Dogs were presented the command of "sit" every $5 \mathrm{sec}$ for $5 \mathrm{~min}$. Sessions were repeated 3 times with 3 min resting periods between each session. In Experiment 1, the dog's daily food was used as the reinforcer. In Experiment 2, praise/stroking were used as the reinforcer. The praise was a verbal "good" while stroking the dog's back gently. Experiment 1 was conducted on all dogs first, then after 2 to 3 weeks, Experiment 2 was performed. The experiments were conducted in the dog's familiar exercise yard by a handler (male, 29 years, and accustomed to working with dogs). The handler was not a stranger to any of the dogs, but only made contact with dogs during the experiments. When a dog responded to a command correctly, the handler gave the dog a reinforcer immediately. When the dog reacted incorrectly or did not show any response, the handler did nothing until next command.

Behavioral evaluation: Whole sessions were recorded using a digital video camera (GSC-R60, Toshiba, Japan). The number of serial operant sequences of administration of the command, response to the command and delivery of reinforcer were counted as the frequency of correct responses to the command. The period (seconds) during which each dog gazed at the handler, which indicated dog's concentration, was also measured.

Statistical analysis: The correlation between the frequency of correct responses to the commands and the length of time spent gazing at the handler was calculated using the Pearson product-moment correlation coefficient or the

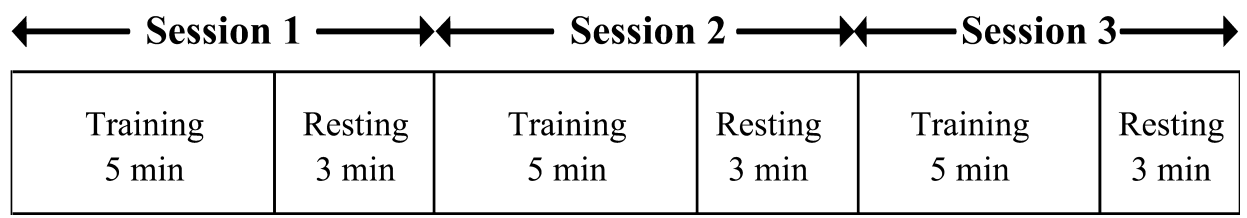

Fig. 1. Experimental procedure. Dogs were given the command to sit every five seconds for five minutes. In Experiment 1, food was used as a reinforcer. In Experiment 2, praise/stroking was used as a reinforcer. 


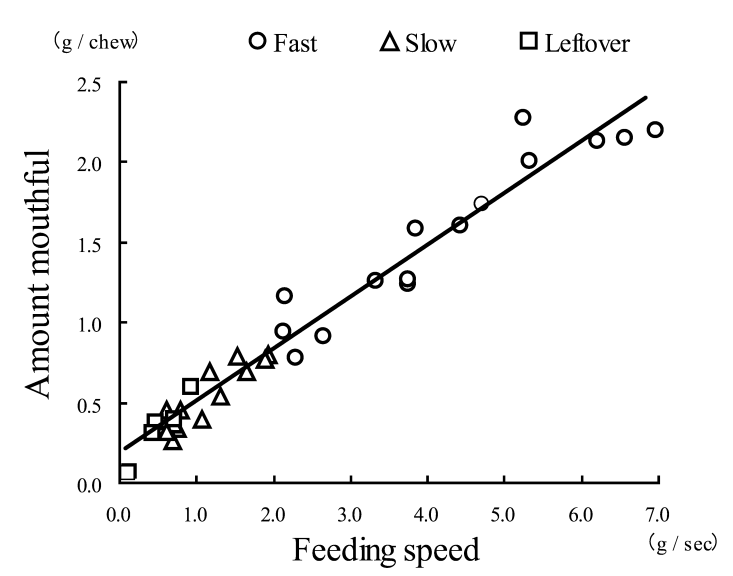

Fig. 2. Relationships between the feeding speed and the amount of food per mouthful for each dog. There was a significant positive correlation between them (Spearman rank correlation coefficient: rank correlation $=0.8, p<0.05$ ). Circles: Fast group; triangles: Slow group; squares: Leftover group.

Spearman rank correlation coefficient. The differences among sessions in each group were analyzed using Bonferoni/Dunn test in one-way factorial ANOVA. The differences between reinforcers were analyzed by Student's $t$ test and $p$ values $<0.05$ were considered significant using the average of three sessions. Results are expressed as means \pm SE.

\section{RESULTS}

According to feeding patterns, the thirty-four dogs were divided into three groups: Fast $(\mathrm{n}=15)$, Slow $(\mathrm{n}=13)$ and Leftover $(\mathrm{n}=6)$. There was no distribution bias according to the facilities (data not shown). Feeding speed did not correlate significantly with the body size, in other words, with the length and depth of the muzzle of the dog (data not shown). Relationships between the feeding speed and the amount of food per mouthful for each dog were shown in Fig. 2. There was a significant positive correlation between them $(\mathrm{rs}=0.8$, $p<0.05$ ). Figure 3 shows the comparison of the frequency of correct responses to the commands and the length of time spent gazing at the handler in Session 1 using food (Experiment 1, Fig. 3-a) or praise/stroking (Experiment 2, Fig. 3-b) as the reinforcer. There were significant positive correlations between the frequency of correct responses and the length of time spent gazing at the handler ( $\mathrm{rs}=0.9$ (a) and 0.8 (b), $p<0.05$ ).

The comparison among groups in each session for Experiment 1 is shown in Fig. 4. Over all of the sessions, the Fast group showed significantly longer periods of gazing at the handler than the Slow and Leftover groups $(p<0.05)$. During Sessions 1 and 3, the Slow group exhibited a longer duration of gazing at handler than the Leftover group $(p<0.01)$. The Fast and Slow groups correctly responded to the command more frequently than the Leftover group
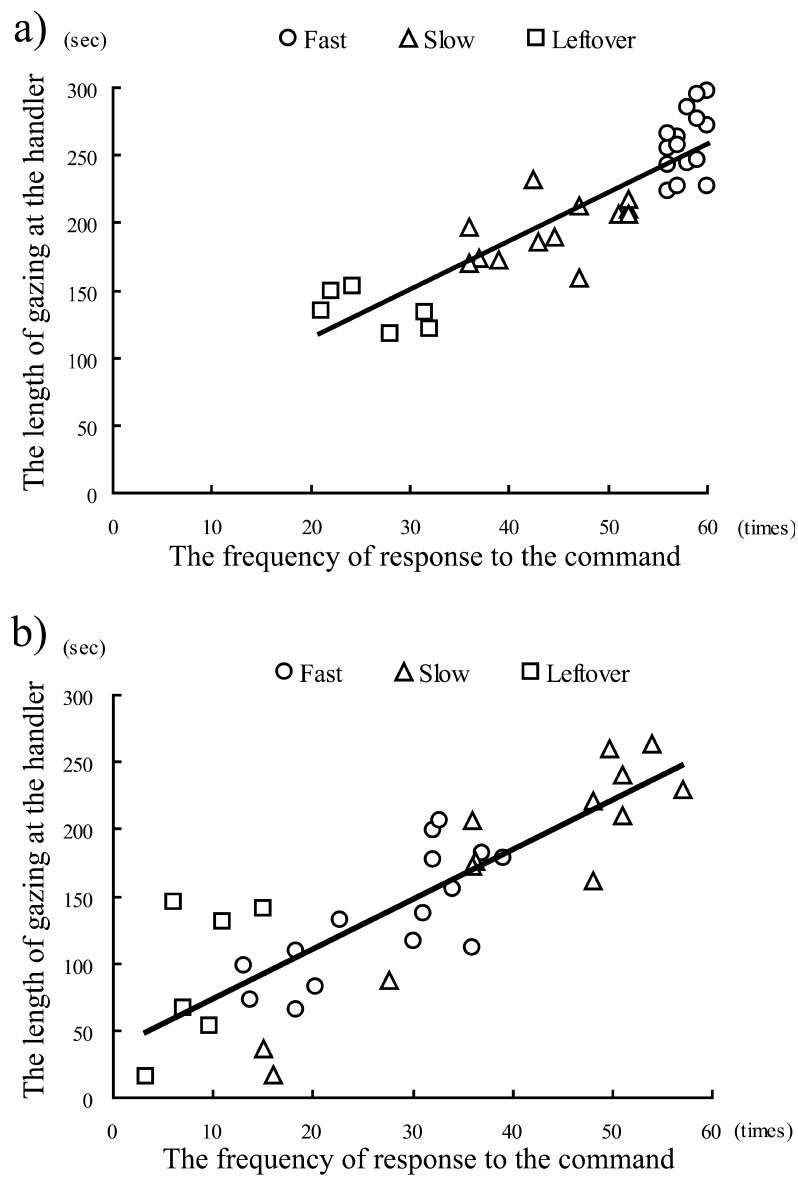

Fig. 3. The comparison between the frequency of correct responses to the commands and the length of time spent gazing at the handler during Session 1: a) food was used as a reinforcer (Pearson product moment correlation coefficient: rank correlation $=0.9, p<0.05)$, b) praise/stroking was used as a reinforcer (Spearman rank correlation coefficient: rank correlation $=0.8$, $p<0.05)$. Circles: Fast group; triangles: Slow group; squares: Leftover group.

throughout the sessions $(p<0.05)$.

The results of Experiment 2, using praise/stroking as the reinforcer, are shown in Fig. 5. The Slow group showed significantly longer periods of gazing at the handler than the Leftover group $(p<0.05)$. The frequency of correct responses to the command in the Slow group was significantly higher than that for the Leftover group $(p<0.05)$.

In the Fast and the Leftover groups, the average period of gazing (sec) at the handler of all sessions showed significant differences between reinforcers [food $v s$. praise/stroking: $253.1 \pm 1.9$ vs. $121.7 \pm 21.7, p<0.01$ (Fast group); $99.5 \pm$ 12.9 vs. $35.2 \pm 19.7, p<0.05$ (Leftover group)]. The frequency of correct responses (number) to the commands also differed significantly between reinforcers [food $v s$. praise/ stroking: $54.5 \pm 0.4$ vs. $26.1 \pm 4.4, p<0.01$ (Fast group); 21.3 \pm 2.2 vs. $6.5 \pm 3.0, p<0.05$ (Leftover group)]. The Slow group did not show significant differences between rein- 

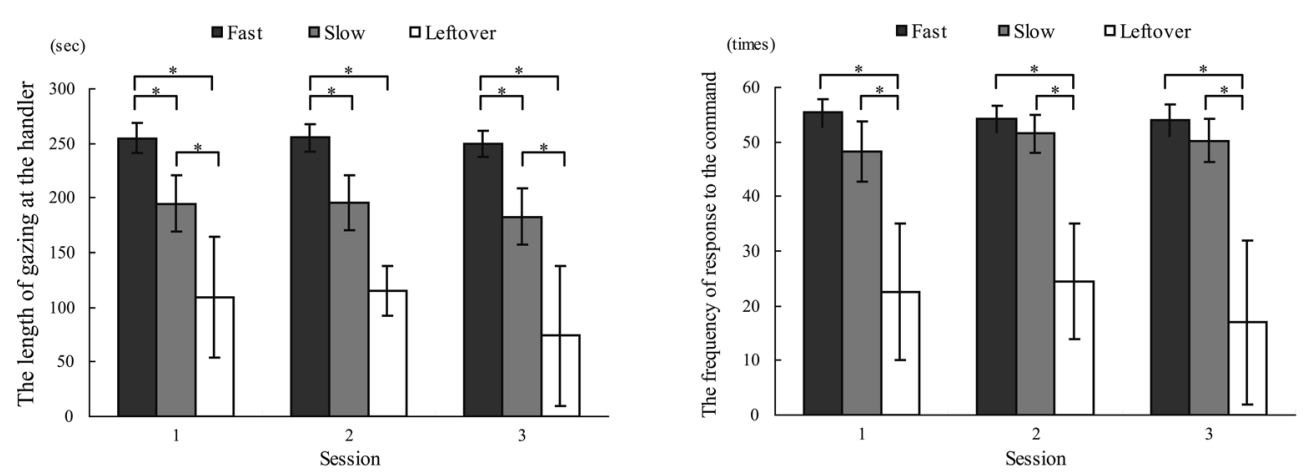

Fig. 4. The comparison among groups in each session of the length of time spent gazing at the handler (a) and the frequency of correct responses to the command (b) in Experiment 1. ${ }^{*} p<0.05$ by Bonferoni/Dunn test in one-way factorial ANOVA.
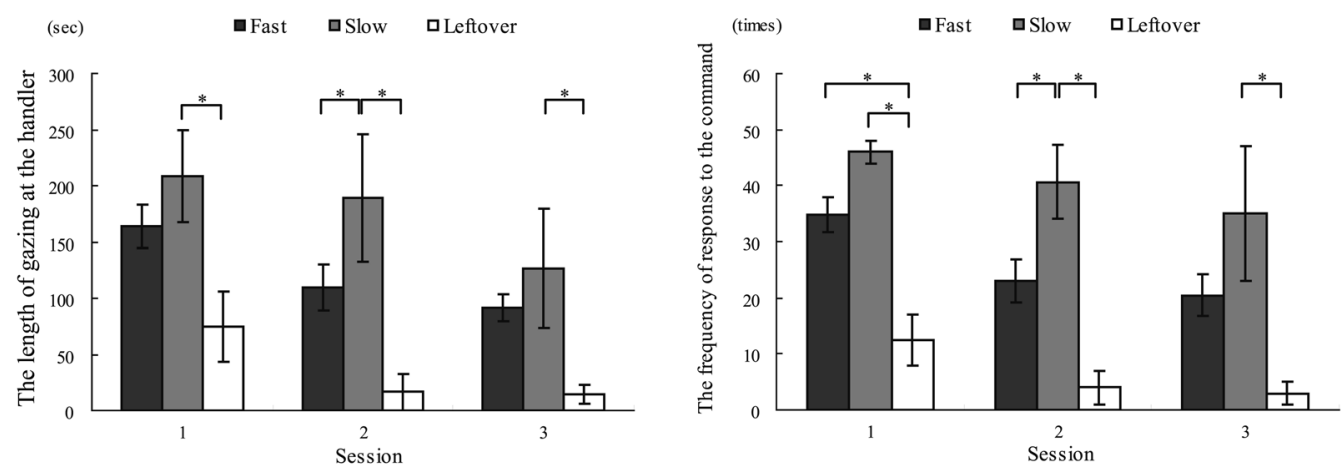

Fig. 5. The comparison among groups in each session of the length of time spent gazing at the handler (a) and the frequency of correct responses to the command (b) in Experiment 2. ${ }^{*} p<0.05$ by Bonferoni/Dunn test in one-way factorial ANOVA.

forcers on the period (sec) (food $v s$. praise/stroking: $191.3 \pm$ $4.1 v s .174 .4 \pm 24.7$ ) and the frequency (number) (food vs. praise/stroking: $50.0 \pm 0.9$ vs. $40.6 \pm 3.2$ ).

\section{DISCUSSION}

The present study demonstrates a correlation between dogs' responses to commands and their feeding patterns. As shown in Table 1 , the feeding speed $(\mathrm{g} / \mathrm{sec})$ and the amount of food consumed per mouthful did not depend on sex, age, or the dogs' body size. On the whole, there was a significant positive correlation between the frequency of correct response to the command and the length of time that a dog spent gazing at the handler, regardless of the reinforcer. In Experiment 1, dogs in the Fast group, which did not chew every mouthful, maintained high scores, even when the session was repeated. This suggests that the Fast group could maintain the operant response to the command when food was used as reinforcer. On the other hand, it also suggests that Fast group did not associate the primary reinforcer (food) with the secondary reinforcer (praise/stroking), since these dogs showed significantly lower scores when using praise/stroking. In Experiment 2, their responses were reduced from Session 1 to Sessions 2 and 3. Ten out of fif- teen in this group were breeds that are vulnerable to obesity $[4,7]$. These data suggest that owners of these breeds should be cautious about using food as the reinforcer in training because of their high level of interest in food and the excess intake of calories.

The Slow group, which achieved almost the same scores as the Fast group with respect to the frequency of correct response to the command, did not show any difference when praise/stroking was used as reinforcer. This group sustained appropriate responses to the command when praise/stroking was used as the reinforcer. This result suggests that the Slow group could take food as the primary reinforcer and then associate that food with praise/stroking as the secondary reinforcer. Furthermore, it is suggested that this group may be able to connect the reward to the work with the handler, and consider this work to be pleasant. A scheme for motivation of the Slow dogs is proposed to be 'command=food=handler'. Therefore, we suggest that the Slow group can be motivated by a wide variety of stimuli during training with positive reinforcement. We predict that these dogs would be easy to handle for the typical dog owner.

The Leftover group showed consistently low responses to food and praise/stroking, and these reinforcers could not motivate these dogs. Therefore, this study found that food 
might not be an adequate reward for this group. These dogs appear to have difficulty connecting the reward with the secondary reinforcer. The Leftover group consisted of three ancient breeds, Greyhound, Pyrenean Mountain Dog, and Vizla. Although further studies are required, the process of living with humans might have affected the feeding pattern and interest in food of these dogs. Concerning the analyses of training methods used on working dogs, Haverbeke et al. [5] suggested that motivationally equivalent rewards might need to be identified and given to dogs instead of food (e.g. tug and retrieve games). Furthermore, Reid [8] insisted that in any training situation, one must consider the motivational state of the animal as well as the learning contingencies, and the potential for competing motivations.

Even though there were some differences, all groups showed lower scores for praise/stroking than those for food. Secondary reinforcement is acquired through experience [6], therefore all groups showed low responses to praise/ stroking. But perhaps we would have gotten different results if the experiments were conducted by facility staff or repeated by us. Although there might be the possibility that the breed difference such as trainability, friendliness to human, and sensitivity to stimuli affect dog's responses to commands, the further research would be needed in more numbers of individual breeds.

In this study, identification of a dog's feeding pattern was a simple and effective way to evaluate its level of interest in food. This may contribute to the selection of the optimum reinforcer for each dog. Using the optimum reinforcer should lead to better results in training and consequently a better relationship between the dog and its owner.

ACKNOWLEDGMENT. This study was partially supported by a project grant (Creative Research Project, 2008) awarded by the Azabu University Research Services Division.

\section{REFERENCES}

1. Bradshaw, J. W. S. 2006. The evolutionary basis for the feed- ing behavior of domestic dogs (Canis familiaris) and cats (Felis catus). J. Nutr. 136: 1927S-1931.

2. Clutton-Brock, J. 1995. Origins of the dog: domestication and early history. pp. 7-20. In: The Domestic Dog: Its Evolution, Behaviour and Interactions with People (Serpell, J. ed.), Cambridge University Press, Cambridge.

3. Colwill, R. M. and Rescorla, R. A. 1990. Evidence for the hierarchical structure of instrumental learning. Anim. Learn. Behav. 18: 71-82.

4. Edney, A. T. and Smith, P. M. 1986. Study of obesity in dogs visiting veterinary practices in the United Kingdom. Vet. Rec. 118: 391-396.

5. Haverbeke, A., Laporte, B., Depiereux, E. and Giffroy, J. M. and Diederich, C. 2008. Training methods of military dog handlers and their effects on the team's performances. Appl. Anim. Behav. Sci. 113: 110-122.

6. Herrnstein, R. J. 1964. Secondary reinforcement and rate of primary reinforcement. J. Exp. Anal. Behav. 7: 27-36.

7. Lund, E. M., Armstrong, P., Kirk, C. and Klausner, J. 2006. Prevalence and risk factors for obesity in adult dogs from private US veterinary practices. Intern. J. Appl. Res. Vet. Med. 4: 177.

8. Reid, P. J. 1996. Excel-erated learning, James \& Kenneth Publishers, Berkeley.

9. Skinner, B. F. 1935. The generic nature of the concepts of stimulus and response. J. Gen. Psychol. 12: 40-65.

10. Skinner, B. F. 1950. Are theories of learning necessary? Psychol. Rev. 57: 193-216.

11. Thorndike, E. L. 1927. A fundamental theorem in modifiability. Proc. Natl. Acad. Sci. U.S.A. 13: 15-18.

12. Throne, C. 1995. Feeding behaviour of domestic dogs and the role of experience. pp. 103-114. In: The Domestic Dog: Its Evolution, Behaviour and Interactions with People (Serpell, J. ed.), Cambridge University Press, Cambridge.

13. Tolman, E. C. and Hpnzik, C. H. 1930. Introduction are removal of reward, and maze performance in rats. Publ. Psychol. 4: 257-275.

14. Vila, C., Savolainen, P., Maldonado, J. E., Amorim, I. R., Rice, J. E., Honeycutt, R. L., Crandall, K. A., Lundeberg, J. and Wayne, R. K. 1997. Multiple and ancient origins of the domestic dog. Science 276: 1687-1689.

15. Wayne, R.K. 1993. Molecular evolution of the dog family. Trends Genet. 9: 218-224. 